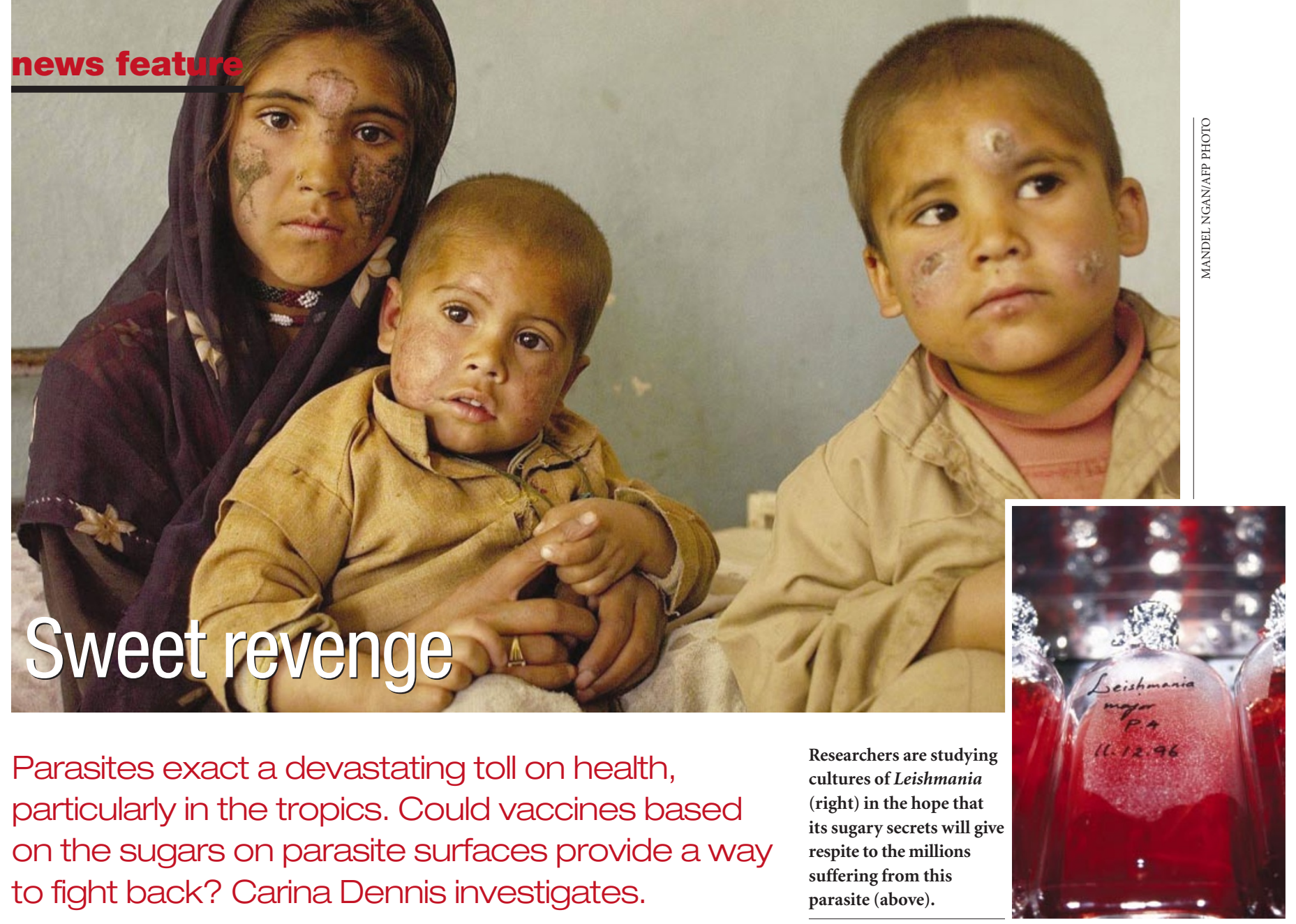

T hey are the undercover agents in the army of pathogens that continually assaults our bodies — parasites that live inside us for months or even years, constantly dodging a gradually tiring immune system, and even burrowing inside our cells.

Various species of Leishmania, for instance, can even hide inside some of the immune cells that are supposed to destroy foreign invaders - surviving unscathed in a sac of digesting enzymes that is the cellular equivalent of an acid bath. Leishmania causes disfiguring skin sores, and can also result in anaemia, fever and swelling of the liver and spleen. Some 12 million people, mostly in the tropics and subtropics, are currently affected.

Faced with such formidable opponents, vaccine developers have so far drawn a blank - there is currently no vaccine on the market that provides full protection against single-celled protozoan parasites such as Leishmania. "Vaccine candidates that work well in animal models have been disappointing in human trials," observes David Sacks, head of intracellular parasite biology at the US National Institute of Allergy and Infectious Diseases in Bethesda, Maryland.

Part of the problem is that many parasites are able to shuffle their surface proteins rapidly, thereby escaping recognition by the immune system. Trypanosoma brucei, a protozoan that causes African sleeping sickness, is the ultimate master of disguise, living in

the bloodstream and replacing its coat of proteins every two weeks.

Given such constantly shifting targets, it is perhaps unsurprising that conventional approaches to immunization — involving whole, killed organisms or purified surface proteins - have yielded little success. So some researchers are now plotting an alternative line of attack: they are trying to get the immune system to respond not to proteins, but to the complex sugars that parasites carry on their surfaces. "Carbohydrates have untapped potential," says Mitch Kronenberg, an immunologist at the La Jolla Institute for Allergy and Immunology, part of the University of California, San Diego.

\section{Sugar rush}

This new focus on complex sugars as candidate vaccines is part of a wider upsurge of interest in glycobiology - the study of the roles played by such molecules in biological systems. Most of the sugars found on cells' surfaces are linked to proteins or lipids, to form glycoproteins or glycolipids, respectively (see diagram, opposite). Over the past few years, these complex molecules have been found to play crucial roles in important biological processes, including cell communication and signalling.

Although the research is in its infancy, there are good reasons to be cautiously optimistic about a sugar-based approach to vaccine development. Parasites carry sugars that are distinct from those of their hosts - a basic requirement for any type of candidate vaccine molecule. And carbohydrates have an advantage over proteins in that they are less changeable. Many complex sugars are elaborately branched molecules, the construction of which depends on labyrinthine biochemical pathways involving many different enzymes. Whereas a new protein can be produced without major genetic upheaval, that's not usually true for a complex sugar. "Carbohydrates are more evolutionarily stable," says Kronenberg. "Parasites can't change them very easily."

What's more, targeting sugars is appealing because they seem to be central to many parasites' ability to conquer the host's defences. "Parasites are very dependent on carbohydrates for their survival and infectivity," says Michael Ferguson, a molecular parasitologist at the University of Dundee, UK. The sugary coats of many parasites, he points out, protect them from harsh environments, help them to invade host cells, and allow them to evade surveillance by the immune system. For example, the surface sugars carried by Leishmania are thought to help to protect it from being disintegrated by the enzymes of the intracellular sac in which it resides.

The principle of sugar-based vaccines has already been demonstrated, albeit with lessevasive bacterial infections. Vaccines based on 


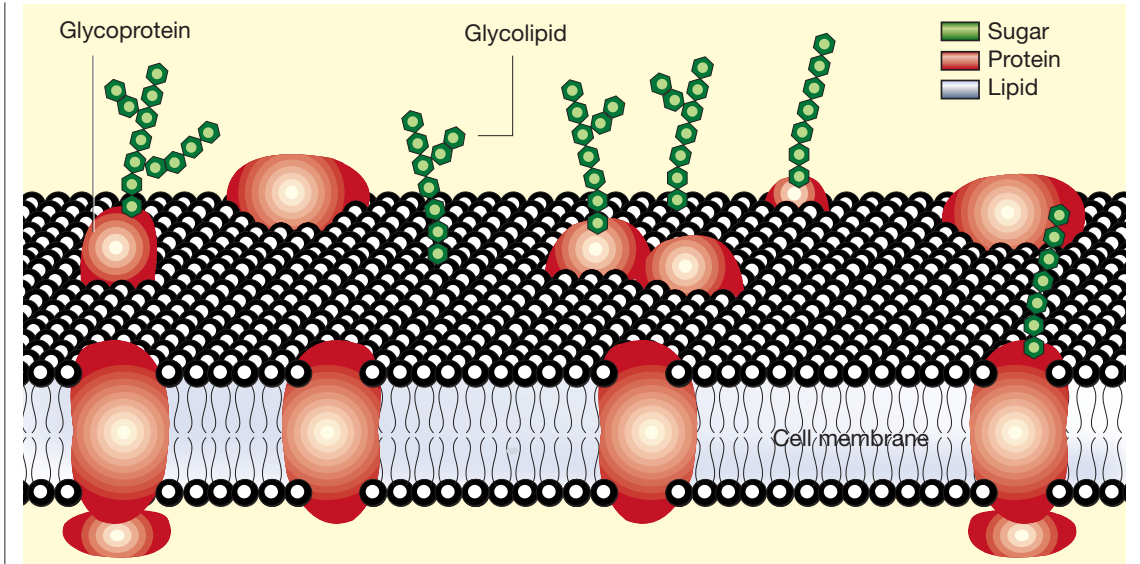

Many proteins and lipids on the surfaces of cells are tagged with sugars to form glycoproteins and glycolipids. Some of these complexes have important functions in cell communication and signalling.

sugars from the surface coats of Streptococcus pneumoniae, which causes pneumonia in young children, and Haemophilus influenzae type $b$, which causes a form of meningitis that can result in deafness, brain damage or death, are already widely used. And some early studies provided encouraging signs that a similar approach might work for protozoan parasites — for instance, a glycolipid called lipophosphoglycan (LPG), found on the surface of Leishmania, was shown in the mid-1980s to protect mice from subsequent infection ${ }^{1}$.

But researchers striving to develop vaccines against parasites have only recently begun to pay serious attention to sugars. The explanation is partly cultural: "Carbohydrates just aren't as flashy as proteins," says Sam Turco, a glycobiologist at the University of Kentucky in Lexington.

Technical challenges have also caused sugar vaccines to lag behind their protein counterparts. The biggest problem is that our immune system is not very good at recognizing sugars - and is even worse at remembering them. In contrast, it never forgets a foreign protein, and mounts a more vigorous response the next time that protein is encountered. For this reason, proteins have always been preferred as vaccine candidates.

Our immunological memory depends on the immune system's T cells, which tend to ignore sugars. But this hurdle can be overcome simply by linking the sugar to a protein. "People tend to shy away from carbohydrates as vaccines because of the widespread dogma that they aren't very immunogenic," says Richard Cummings, a glycobiologist at the University of Oklahoma Health Sciences Center in Oklahoma City. "But a carbohydrate can be made very immunogenic with a protein attached." In the case of the successful bacterial version of the protein toxins produced by the bacteria that cause tetanus or diphtheria.

Scientists have also been deterred from working on carbohydrates because of the molecules' complexity, which makes them culture to squeeze out just a few milligrams vaccines, the sugar is linked to an inactivated difficult to synthesize in the lab and difficult to analyze structurally. Whereas the subunits of DNA and protein are strung together in a linear way, like beads on a necklace, carbohydrate subunits can attach to one another at many different points, much as a child's building blocks can fit together to form a vast assortment of configurations.

\section{High volume}

It is also notoriously difficult to extract sufficient quantities of a parasite's carbohydrate to study. Until relatively recently, researchers had to grow huge vats of parasite cultures just to be able to extract enough of the sugar they were interested in. "That's why you had graduate students," quips Turco, who vividly recalls preparing tens of litres of Leishmania of a particular glycoprotein or glycolipid.

Now, however, advances in the sensitivity of analytical techniques such as mass spectrometry and nuclear magnetic resonance have allowed sugars to be characterized from much smaller amounts of sample material. "Previously, we'd start with the easiest things first, which meant solving the structures of the most abundant carbohydrates," says Ferguson. "But as the instrumentation improves, we can tackle the structures of minor cell-surface carbohydrates, which are exquisitely important."

In parallel, improved methods for making sugars have also made it easier for researchers to study and evaluate vaccine candidates. After determining a sugar's structure, they no longer have to obtain further samples by purifying the sugar from the parasite itself which may be difficult to grow in the laboratory. Rather, the sugars can be synthesized artificially. And at the Massachusetts Institute of Technology in Cambridge, a team led by organic chemist Peter Seeberger has streamlined the process

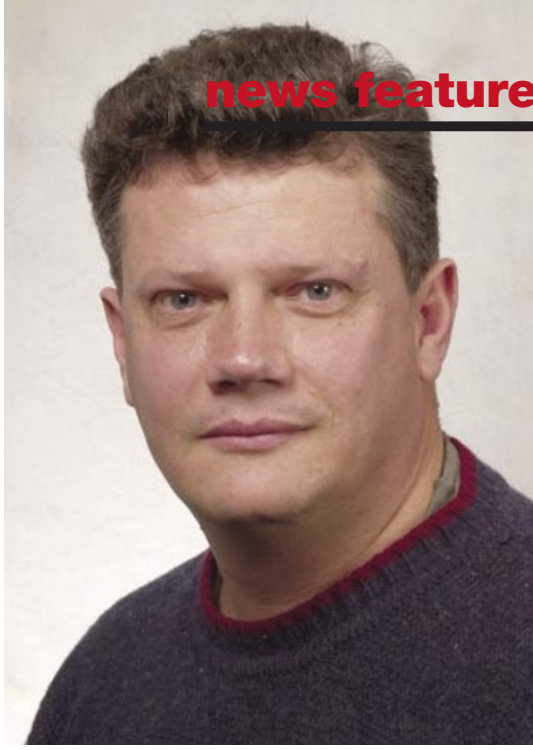

Sugar craving: Louis Schofield wants to develop a carbohydrate-based vaccine to combat malaria.

by modifying an automated peptide synthesizer. "The hardware is the same, just the chemistry has changed," he says. "Carbohydrates that once took years to construct can now be created in a matter of days."

The device that Seeberger modified is normally used to create specific peptides - the subunits from which complex proteins are constructed - by adding amino acids in succession to form a chain, tethered to a polystyrene bead. Using their adapted device, Seeberger and his colleagues can create complex branched sugars from smaller sugar molecules, determining where each new molecule is added by masking other sites to which it might link with 'protecting' chemical groups ${ }^{2}$.

Louis Schofield, a parasite immunologist at the Walter and Eliza Hall Institute of Medical Research in Melbourne, Australia, is now working with Seeberger to develop a sugarbased vaccine to curb the impact of and the world's deadliest parasite, Plasmodium falciparum, which is responsible for more than a million deaths every year from

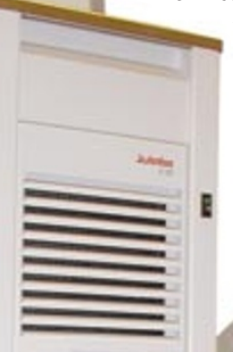

This former protein synthesizer has been modified to build bespoke sugars. 
